\title{
Missing cycles: Effect of climate change on population dynamics
}

\author{
JANAKI BALAKRISHNAN ${ }^{1, *}$, SUDHARSANA V. IYENGAR $^{1}$ and JÜRGEN KURTHS ${ }^{2}$ \\ ${ }^{1}$ School of Natural Sciences and Engineering, National Institute of Advanced Studies (N.I.A.S.), Indian Institute \\ of Science Campus, Bengaluru 560 012, India. \\ ${ }^{2}$ Potsdam Institute for Climate Impact Research, PO Box 601203, Potsdam 14412, Germany. \\ *Corresponding author. E-mail: janaki05@gmail.com
}

\begin{abstract}
Explaining observations in the population dynamics of ecosystems influenced by the environment and being able to correctly predict future events in these complex systems is one of the major challenges in mathematical modelling. We present our models having climate parameters which explain experimental observations of the cyclic population dynamics of the larch budmoth - an insect pest which causes massive defoliation of entire larch forests at high altitudes around the world. The tritrophic ecological system consists of the larch trees, the budmoth and parasitoid species which live off the budmoths. We make an important modification of the models we had proposed earlier by incorporating a slow time dependence in one of the species-specific parameters, in order to provide an explanation for the irregular larch budmoth cyclic outbreaks observed in the French Alps.
\end{abstract}

Keywords. Population cycles; ecological modelling; bursting; bifurcation; coexistence; oscillation death; chaos.

PACS Nos 05.45.-a; 05.45.Pq; 87.23.Cc

\section{Introduction}

Occurrence of cycles in the populations of any species has been a subject of great interest since a long time (see for instance, [1-6]). In certain species this is attributed to prey-predator dynamics such as in hare-lynx and vole-weasel populations. The reasons for occurrence of population cycles in many other species such as lemmings, cicadas (where the cycles are almost always prime numbers), some forest insects such as spruce budworm, gypsy moth, western tent caterpillars are still not fully clear and are being debated [3-6].

The larch budmoth Zeiraphera diniana is an insect pest which infests larch trees at high altitudes around the world and causes destruction of large forest areas by rapid defoliation $[7,8]$. It exhibits population cycles which occur with great regularity; the longest ever recorded time series of population cycles is of the larch budmoth. As many as 123 outbreaks in a span of 1200 years have been documented by direct observations and via dendrochronological studies [9], in the Engadine Valley in the Swiss Alps having a dominant frequency of 9 years and occurring at altitudes of 1500 $2000 \mathrm{~m}$ above sea level. Surprisingly however, after centuries of regular outbreaks, the cycles stopped after 1981 and did not recur after that $[9,10]$. It has been hypothesized that global warming has led to the collapse of the cycles [10]. Variations from the 9-year periodicity of the larch budmoth (LBM) population cycle are known to occur elsewhere [7,11,12]. Another intriguing fact is the complete absence of LBM cycles in the Tatra mountains in the Slovakian Carpathians, not too far from the Swiss Alps [7,11].

When temperatures increase after winter, and the larch commences its new foliage with bud-bursts, budmoth larvae emerge from eggs laid by the adult moths on the trees. The larvae begin their wasteful feeding on the foliage and rapidly defoliate vast areas of the larch forests, lending it a burnt and scorched appearance $[7,8]$.

The budmoth infestation thus has an adverse effect not only on the tourism industry, but also on the country's economy - its wood is in demand for its waterproof qualities and is useful as a construction material because it is durable, strong and disease-resistant.

The larch budmoth ecosystem is clearly one which bears memory of past events. Infestation of the larch tree by the budmoth results in depletion of its foliage leading to changes in its biomass and its nutritive content. This in turn affects the current health of the plant and the length of the larch needles. Healthier, untouched larch trees have longer needles, their average length being $15 \mathrm{~mm}$ 
[13]. The larch needle length $L_{t}$ at time $t$ can thus be taken as an estimate of the plant's nutritive content or the quality of its health. In practice, the plant quality index (PQI), a dimensionless measure of the needle length and defined as [13]:

$Q_{t}=\frac{L_{t}-15}{15}$

is used to represent the plant's health.

Previous work by Turchin and others [10,13-16] has shown the necessity of including three species in their model to capture and sustain the budmoth population cycles: the larch trees represented by their PQI, the budmoths which infest them, and parasitoids which live off the budmoths. Turchin's tritrophic model $[13,15]$ is able to reproduce the 9-year budmoth cycles seen in the Swiss Engadine valley but can reproduce no other feature mentioned above.

Recently, we proposed a model [17] including climate /environmental factors which not only reproduces the 9-year periodicity for the budmoth population, but also can explain and reproduce variations in the periodicity seen in the cycles, the absence of cycles in the Tatra mountains, and the collapse of the cycles after 1981 in the Swiss Alps.

The budmoth at any given time can be host to more than one species of parasitoids. In fact, about 94 species of parasitoids are known which can attack the larch budmoth [14]. In a more recent work [18] we modified our model to include the presence of more parasitoids and found that this inclusion provides an alternative explanation for the collapse of cycles and changed periodicities.

In the French Alps, outbreaks of the larch budmoth have been recorded for the period 1700-2010 [12] by dendrochronological analyses and observations in the Upper Maurienne Valley in Savoy. During this period this region has been subject to a lot of human intervention - there have been frequent changes in the forest cover because of logging for timber and fuel wood. The area has also seen an expansion of grassland for supporting livestock. Of the 23 budmoth outbreaks in this time, the dominant outbreak frequencies are 10 and 15 years, followed by periodicities of $8,13,18$ and 26 years, each cycle lasting for $2-5$ years. There is no model in the literature so far which can capture the irregularities and variations in the periodicities, nor the differing lengths of the cycles.

In this current work we show that by making a small but significant modification of our models proposed in our previous work [17-19], we can qualitatively capture nearly all the features of the budmoth outbreaks in the French Alps.

\section{A tritrophic model}

Turchin's tritrophic model [15] which reproduces the 9-year periodicity is given by the following set of equations for the population densities of the budmoth $N_{t}$ and parasitoids $P_{t}$ and the plant quality index $Q_{t}$ at any given time $t$ :

$$
\begin{aligned}
N_{t+1} & =\lambda N_{t} \frac{Q_{t}}{\delta+Q_{t}} \exp \left[-\beta N_{t}-\frac{a P_{t}}{1+a w P_{t}}\right] \\
P_{t+1} & =b N_{t}\left\{1-\exp \left[-\frac{a P_{t}}{1+a w P_{t}}\right]\right\} \\
Q_{t+1} & =(1-\alpha)\left(1-\frac{N_{t}}{\gamma+N_{t}}\right)+\alpha Q_{t} .
\end{aligned}
$$

Here, $\lambda$ denotes the budmoth intrinsic growth rate, $a$ is the parasitoid searching rate, $\beta$ denotes the budmoth intraspecific competition, $c$ is the half saturation constant for the budmoth uptake, $\delta$ stands for the half saturation constant for the PQI, $b$ indicates the number of viable parasitoid offspring produced at an infested budmoth site per generation and $w$ is the parasitoid wasting time whose value ranges between 0 (when no time is wasted between 2 encounters with its prey) and $\infty$ (when the parasitoid is unable to find its prey). The quantity $\alpha$ represents the plant vulnerability to budmoth attacks and in some way imbibes memory of the previous growth of the plant. Thus it contains information of the nutrient content of the plant biomass at the end of the previous infestation. Its value ranges from 0 to $1 ;(1-\alpha)$ represents the plant recovery.

The dynamics of the budmoth population is modelled with a Nicholson-Bailey interaction term [20] with the parasitoids. A Holling type-2 function [21] for densitydependent feeding is multiplied by $\lambda$ to incorporate varying growth rate. In another variant of this model [13], the intrinsic growth rate of the budmoth was dynamically generated. In both models of Turchin, the choice of the parameters is very tight and in this sense the models are not robust enough - even tiny variations of the parameters within the error bars give cycles with very different periodicities.

\section{3. q-Deformations}

In this system, the memory of past events plays an important role in its future evolution. Growth and spread of larch foliage depends upon the severity of past budmoth infestation and the extent of the plants' recovery from it which in turn depend also upon the extent of consumption of the budmoth larvae by parasitoids, the 
availability of nutrients and favourable environmental conditions such as precipitation and sunshine during the initial leafing period. Since the memory of past parasitoid and budmoth population densities, past PQI and available resources contribute to the current probability distribution, all states of the system are no longer equiprobable - some events are more likely to occur than some others. A Boltzmann-Gibbs distribution which assigns equal probability to all events no longer applies. Such situations are better explained using a Tsallis probability distribution [22] which introduces a parameter $q$. Therefore, instead of considering the system as an ergodic one, we consider it as a $q$-deformed system in which, depending upon the value of $q$, probabilities of occurrence of some events are enhanced, while some others are suppressed. The $q$ value decides whether the system's behaviour is manifested through rare events or through common events. The Boltzmann-Gibbs distribution is recovered in the limit $q \rightarrow 1$.

The idea of generalizing numbers and functions by deforming them by a parameter $q$ dates back to the work of Euler [23] and Heine [24] and later Jackson [25,26]. A simple example is used to illustrate the concept of $q$-deformation. The differential equation $\frac{d y}{d x}=y$ is solved by $\ln y=x$ or $y=e^{x}$. But the solution of the differential equation $\frac{d y}{d x}=y^{q}$ is given by: $y=[1+(1-q) x]^{\frac{1}{1-q}} \equiv e_{q}^{x}$ or: $x=\frac{y^{1-q}-1}{1-q} \equiv \ln _{q} y$. This shows that $y=e_{q}^{x}$ and $x=\ln _{q} y$ can be considered as generalized solutions and are respectively called the deformed exponential and deformed logarithm functions. The original exponential and logarithm functions are recovered when $q \rightarrow 1$. $q$-deformed numbers and functions have uses and applications in explaining experimental observations in different situations [27-34]. $q$-deformed functions and numbers naturally appear with the non-extensive formulation of statistical mechanics of Tsallis and preserve the Legendre transform structure of thermodynamics. The deformation obtained [25] by Taylor-expanding $e_{q}^{x}$ around $x=0: x_{q}=\frac{x}{1+(1-q)(1-x)}=\frac{x /(2-q)}{1+\frac{q-1}{2-q} x} \quad$ was used by Tsallis [22]. We employ this deformation in our work as it mimics the Holling type-2 functional response in ecological systems.

In the model we proposed [17-19], we described the populations in the system using $q$-deformed variables, taking different values of the deformation parameter $q_{i}$ for each species $i$, since each species could respond differently to any given stimulus.

For the parasitoids, we chose $q_{y}=q_{\text {parasitoids }} \neq 1$ (corresponding to the hyperbolic (Holling type-2) functional response) to avoid overcounting of parasitoids which results with the usual assumption made that the budmoth larvae are completely converted into parasitoid density. The actual biological feature of densitydependent removal of foliage (including damaged or partially consumed needles) by the budmoth is captured by taking $q_{z}=q_{\mathrm{PQI}} \neq 1$ [17-19]. This latter feature is captured only in our model and is absent in other models in the literature. We choose $q_{x}=q_{\text {budmoth }}=1$ for simplicity.

\section{4. q-Deformed model with 2 parasitoid species}

In this present work, we try to explain the features observed in the larch budmoth outbreaks in the French Alps - a system made more complex because of human intervention in it. We assume that 4 species are present in the system - the larch tree represented by the PQI $z$, the budmoth population density represented by $x$, and population densities $y$ and $v$ of two species of parasitoids. We assume that though both $y$ and $v$ feed on the budmoth larvae, they do not interact with each other. As reported in our earlier work [18], addition of a fresh parasitoid species into our system of equations increases the time period of the cyclic outbreaks generated by our system, in agreement with observations [8]. Our equations in dimensionless form to describe the system are as follows [18]:

$$
\begin{aligned}
& x_{t+1}=\frac{\rho_{z} z_{t}}{1+\mu_{z} z_{t}} \lambda x_{t} \exp \left(-x_{t}-\frac{\rho_{y} y_{t}}{1+\mu_{y} y_{t}}-\frac{\rho_{v} v_{t}}{1+\mu_{v} v_{t}}\right) \\
& y_{t+1}=c x_{t}\left(1-\exp \left(-\frac{\rho_{y} y_{t}}{1+\mu_{y} y_{t}}\right)\right) \\
& v_{t+1}=\kappa c x_{t}\left(1-\exp \left(-\frac{\rho_{v} v_{t}}{1+\mu_{v} v_{t}}\right)\right) \\
& z_{t+1}=(1-\alpha)\left(1-\frac{x_{t}}{m+x_{t}}\right)+\alpha\left(h-s x_{t}\right) \frac{\rho_{z} z_{t}}{1+\mu_{z} z_{t}} \\
& \mu_{i}=\frac{q_{i}-1}{2-q_{i}}, \quad \rho_{i}=\frac{1}{2-q_{i}}, \quad(i=y, v, z) .
\end{aligned}
$$

The dimensionless variables $x_{t}, y_{t}, z_{t}$ are related to $N_{t}, P_{t}$ and $Q_{t}$ of eqs (3) and (4) in the following way: $x_{t}=\beta N_{t}$, $y_{t}=a P_{t}$ and $Q_{t}=z_{t}$.

The parasitoid efficiency in capturing its host from among all the available hosts is quantified by $c=\frac{a b}{\beta}$ and is an indicator of its performance and of how well its population density increases. The efficiency of the budmoth in consuming the larch needles is measured by the quantity $m=\beta \gamma$. The parameter $\kappa$ is a measure of the relative strength of the second parasitoid with respect 
to the first one; for instance, $\kappa<1$ indicates that $v$ is weaker than $y$. The climate parameters $h$ and $s$ are those which we had introduced in Ref. [17] to incorporate effects of climate / environment on the system.

In an earlier version of our model [19] we introduced the feature of density-dependent removal of the larch needles through the term $\alpha z_{q_{t}} x$ (in eq. (8)) in place of their constant removal which is in Turchin's models.

In that work [19] we had related the $q$-deformed numbers to physical quantities: $q_{y}$ to $w: \mu_{y}=\frac{q_{y}-1}{2-q_{y}}=w$ $1 \leq q_{y} \leq 2$, since $\mu_{y}$ varies from 0 to $\infty$. Further, we had related $q_{z}\left(1 \leq q_{z} \leq 2\right)$ to the budmoth intraspecific competition coefficient $\beta$ : $q_{z}=1+\beta$, with $\mu_{z}=\frac{1}{\delta}$ and $\rho_{z}=\frac{1}{\beta \delta}$.

We demonstrated through our model in Ref. [17] that the collapse of the budmoth cycles in the Engadine valley after 1981 was a result of changing climatic conditions including rising temperatures after a tipping point was reached. We also predicted recurrence of the cycles there with a changed periodicity of 40 or 100 years.

The stability analysis for the system in eqs (3-7) was discussed in Ref. [18]. Limit cycles arise in this system via Niemark-Sacker bifurcation with respect to all the parameters [18]. Figure 1 shows cycles for 4 different values of $\kappa$. One can see that whenever the budmoth population density is low, the PQI increases and the parasitoid population densities decrease. As the budmoth population increases, so do the parasitoid populations and the PQI falls. When $\kappa<1$, parasitoid- 1 is stronger and hence the limit cycle is almost in the parasitoid-1 and the LBM plane. As the value of $\kappa$ increases, the

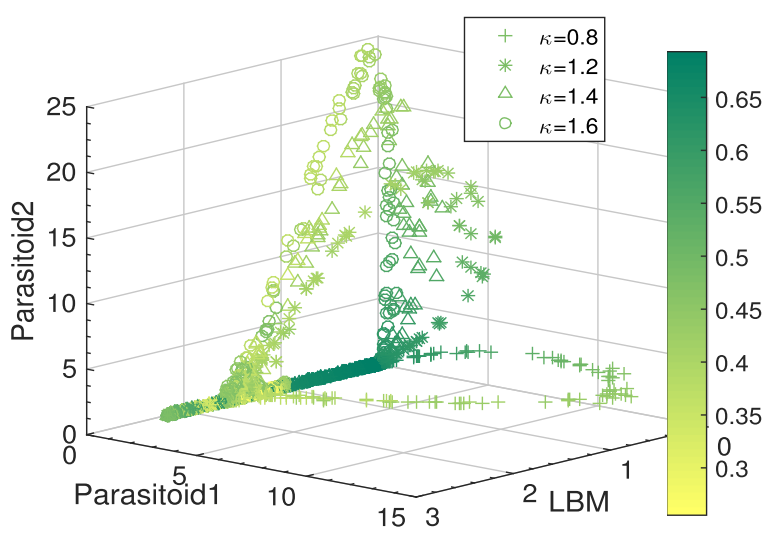

Figure 1. Four-dimensional phase space of the four interacting species generated for 4 different values of $\kappa$. The fourth variable $z$ (the PQI) is shown in colour. Parameter values: $q_{y}=1.13, q_{z}=1.34, \alpha=0.5, m=13, \lambda=8, s=0.5, h=0.5$, $c=12, x_{0}=0.5, y_{0}=1.7, z_{0}=1, v_{0}=0.2$.

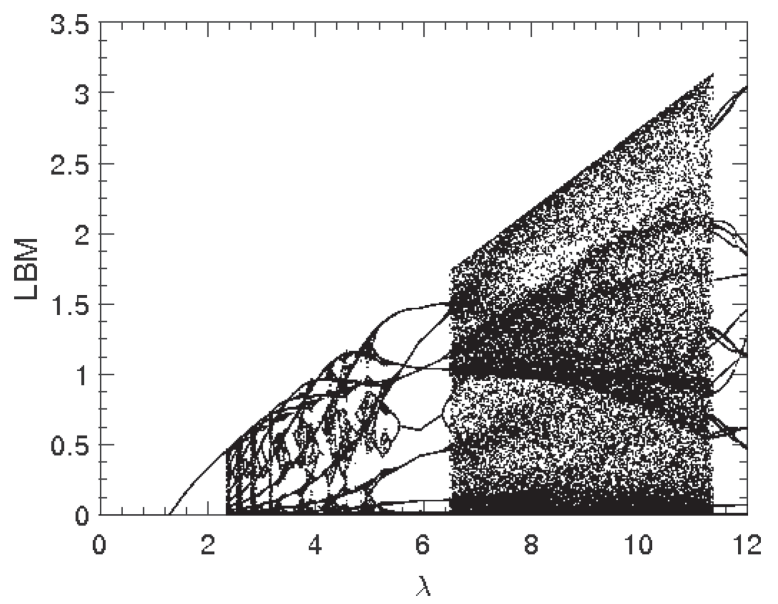

Figure 2. Bifurcation diagram of $x^{*}$ (LBM population density) with respect to the intrinsic growth rate $\lambda$. A NeimarkSacker bifurcation occurs at $\lambda=2.35$. Parameter values: $q_{y}=1.13, q_{z}=1.34, \alpha=0.5, m=13, s=0.5, h=0.5$, $c=12, \kappa=1.6$.

phase portrait shifts from this plane towards the LBM parasitoid-2 plane.

In Ref. [18] we had presented an exhaustive study of the eight-dimensional parameter space of the system with one parasitoid species which shows a very rich dynamical behaviour. All three species, the budmoth, parasitoids and the larch needle lengths show robust cyclic behaviour in certain parameter regimes, and these cycles are always mutually synchronized. This is in agreement with observations; in the Swiss Engadine Valley too which is rich in the time series data of budmoth outbreaks, 9-year periodicity of larch needle lengths was always observed to be synchronized with the 9-year budmoth cycles $[7,8,10,13]$. In figure 2 , we present a bifurcation diagram of the budmoth population with respect to its intrinsic growth rate $\lambda$. Multiple chaotic bubbles are seen that interlace with each other. At around $\lambda=6.5$ there is an interior crisis and a transition to chaotic behaviour.

\section{Modelling the irregular budmoth cycles in the French Alps}

In our earlier work in Ref. [17] where we used the model in eqs (3-7), but with only 1 parasitoid species, we had ascribed the absence of cycles in the Tatras to the sparse larch tree cover in that region which leads to a high intraspecific competition corresponding to low $c$ values. Also, the Tatras have more cloud cover and the environmental parameters, in particular, $h$ values would be expected to be very different from those in the Swiss Engadine valley. 
Interestingly, our analysis, conclusions and deductions have been validated by the observational work published very recently in Refs $[35,36]$.

As mentioned above, in the French Alps, the gaps between the various outbreaks have varied from 5 years to 26 years. Historical evidence suggests that the larch forest area in this region changed during the last three centuries because of war, logging for timber and use of this land for grazing animals. Because of consequent reduction of forest cover, there have been changes in the frequency of the budmoth outbreaks and its periodicity. It has been observed that in regions with less dense canopy, larch budmoth outbreaks are not sustained [12]. This is because with sparser canopy, the intraspecific competition between the budmoth increases and this invariably affects the cycles.

From the Supplementary Information in Ref. [12], we find that the maximum loss in the forest canopy in the French Alps occurred during the period 1800-1850 after which an initiative was begun to restore forest cover. The increasing forest cover (leading to higher $c$ values) paved the way for longer cycles. This is also evident in their data as there are longer than 20-year cycles after the 19th century: between 1800 and 1850 there are 5 cycles, while between 1900 and 1950 there are only two cycles and during 1900-1996 there are only 6 cycles. Thus improving forest cover decreases intraspecific competition which increases $c$ and leads to creation of longer cycles.

There is also one very important feature which distinguishes budmoth outbreaks in the French Alps from those elsewhere. The outbreaks in the French Alps are not very regular and most of them have peak intensities which last for more than a year - most peak intensities here last between 2 and 5 years [12].

Outbreaks which linger for an extended period of time each time they occur, may be represented by bursting solutions. Bursting oscillations are easily generated in this system when there are at least 2 parasitoids present. We assume that this is the case in the French Alps also. Also, in this region, the cycles occur with much longer time periods. The presence of more than one parasitoid species also increases the time periods of the cycles [18] as required here.

The budmoth intraspecific competition $\beta$ is one factor which is clearly affected by the changes in the forest cover caused by human intervention and we expect $\beta$ to increase. But with the presence of more than one parasitoid species, the number of viable parasitoid offspring produced at an infested budmoth site per generation, $b$, also increases. All these could produce an overall slow increase in $c$, which is therefore no longer a constant, but a function of time. We propose this scenario for explaining the outbreaks observed in the French Alps.
Accordingly we incorporate this change in eqs (3-7) by modifying the constant $c$ by $c_{t}$ and include another equation for the evolution of $c$. We assume that the variation in $c$ in time is linear and very slow:

$c_{t+1}=c_{t}(1+\epsilon t)$

where $\epsilon<<1$.

The numerical simulation of the five-dimensional system in eqs (3-7) and (8) is depicted in the time series for the budmoth population density in figure $3 \mathrm{a}$ and $\mathrm{b}$. To enhance visual clarity and for facilitating understanding of the lengthy time series of 12,000 time steps, we have depicted it using the strips function of MATLAB, routinely used in digital signal processing, which splits the time series into $n$ segments and stacks them on top of each other.
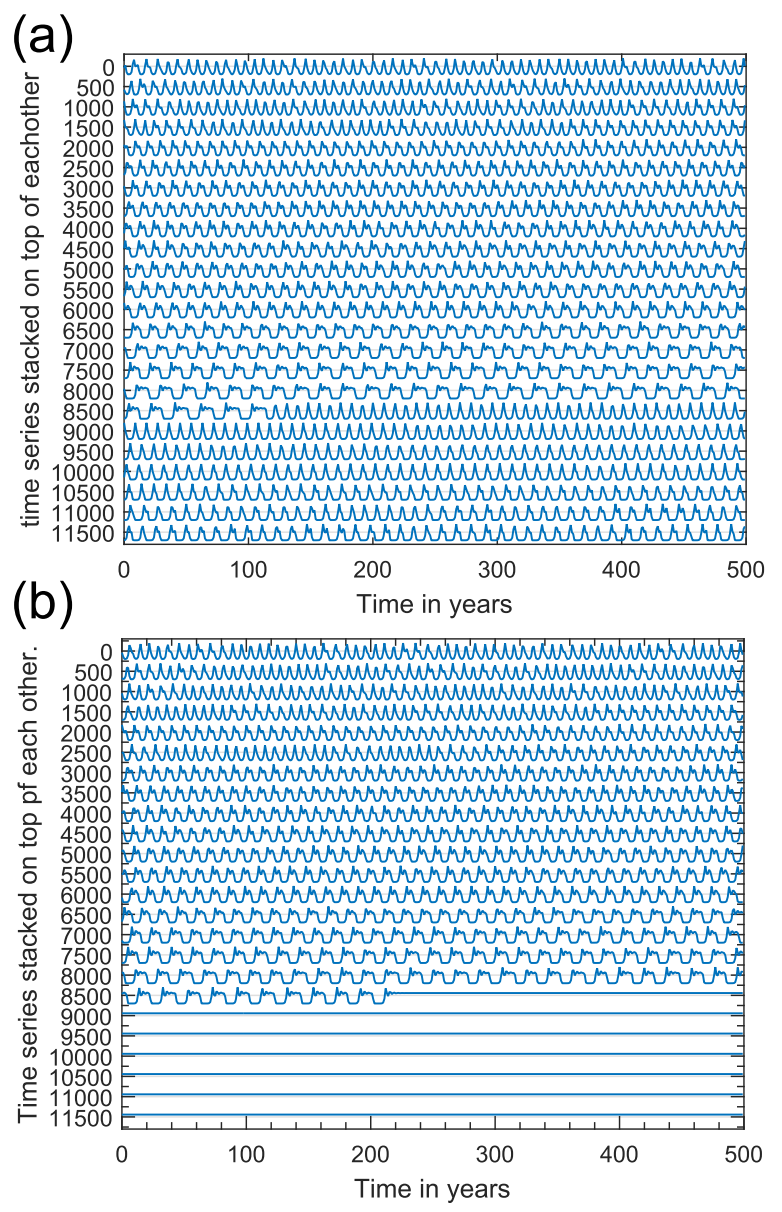

Figure 3. Co-existing solutions of the larch budmoth $x_{t}$ in eqs (3-8). Parameter values: $h=0.5, s=0.5, q_{y}=1.13$, $a=0.5, m=13, q_{z}=1.34, \kappa 1=0.9, \lambda=8, \epsilon=0.00000005$ and $1 \leq c \leq 50$; Initial conditions: $c_{0}=4$, (a): $x_{0}=0.979956195936227, y_{0}=0.480386062641066$, $z_{0}=0.197243808873120, v_{0}=0.256155320585419 ;(\mathbf{b})$ : $x_{0}=0.501608036311145, y_{0}=0.535643466968083, z_{0}$ $=0.743918176543798, v_{0}=0.0961445344243674$. 
We divide the time series into 24 segments, with each segment spanning 500 time steps and stacked on top of each other. The 0 of the time series starts from the topmost left plot and reaches the 500th time step in the topmost right. In the second line plotting is resumed from here and continues till the 1000th time step and so on. As the time series progresses, $c$ is varied according to eq. (8) from 4 to 50 . In this five-dimensional system there is co-existence of attractors (seen in figure $3 \mathrm{a}$ and $\mathrm{b}$ ). There are two stable attractors, one in which bursting gives way to oscillation death for large times and the system settles to a fixed point (figure $3 b$ ) and another in which there is spiking instead (figure 3a). As discussed in Ref. [18] spiking in the budmoth time series for the 2-parasitoid model corresponds to death of one of the parasitoids and is not an interesting situation with regard to the French Alps (since we need at least 2 parasitoids for cycles with longer periods and we need
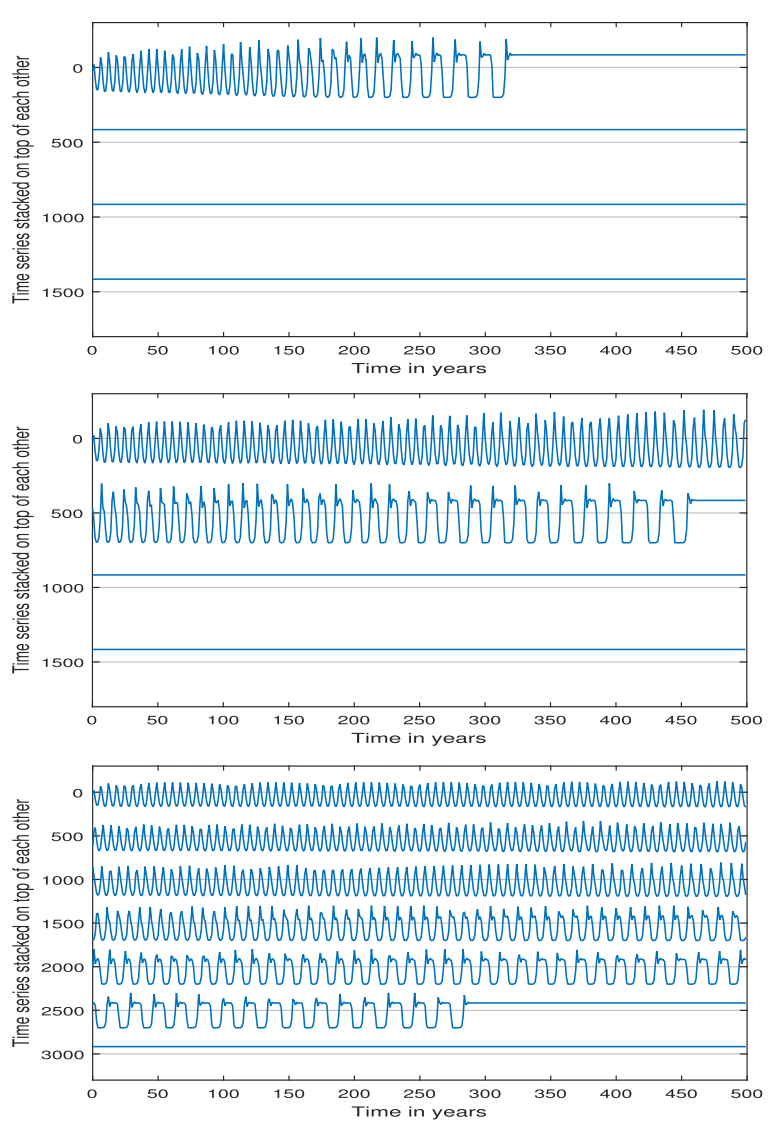

Figure 4. Role of $\epsilon$ in controlling the onset of bursting oscillations. Time series for the larch budmoth $x_{t}$ in eqs (3-8). Parameter values: $h=0.5, s=0.5, q_{y}=1.13, a=0.5, m$ $=13, q_{z}=1.34, \kappa 1=0.9, \lambda=8,1 \leq c \leq 60$; Initial conditions: (same as in figure $3 \mathrm{~b}$ ): $c_{0}=4, x_{0}=0.501608036311145$, $y_{0}=0.535643466968083, z_{0}=0.743918176543798$, $v_{0}=0.0961445344243674$. Top: $\epsilon=0.00005$, middle: $\epsilon=0.000005$, bottom; $\epsilon=0.0000005$. bursting in the budmoth time series to represent longlasting outbreak peaks). The other attractor (figure $3 \mathrm{~b}$ ) which shows bursting behaviour before finally settling to a fixed point is an interesting one.

As $c$ increases, we see in figure $3 b$ that as the spiking behaviour slowly gives way to bursting, the time period of the cycles also increases. When one of the parasitoid species die, the system reverts back to the original cyclic behaviour; otherwise, when both parasitoid species are present, the budmoth population denisty goes into oscillation death and its cycles collapse.

Since changes in $\epsilon$ reflect as slow changes in $c$, it is the initial value $c_{0}$ of $c$ which is primarily important in determining the qualitative change in the system's behaviour. $\epsilon$ plays the role of controlling the onset of the bursting behavior. For higher values of $\epsilon$, the bursting occurs earlier and also, the system undergoes oscillation death earlier. For lower $\epsilon$ values on the other hand, the onsets of both bursting and oscillation death are more delayed. The emergent dynamics (spiking $\rightarrow$ bursting $\rightarrow$ oscillation death or spiking $\rightarrow$ bursting $\rightarrow$ spiking $\rightarrow$ oscillation death) for a given set of initial conditions is however qualitatively the same over orders of magnitude of $\epsilon$. We have depicted this in figure 4 for three representative $\epsilon$ values.

An average of 9 parasitoid species are known to be hosted by a single budmoth in a year in a single site in the Brianconnais region of the French Alps [37]. Our model with more than one parasitoid species is in accord with experimental observations [8].

\section{Comparison and differences between outbreaks in the Tatras and the French Alps}

The larch budmoth cycles were initially modelled using budmoth, PQI and a parasitoid species. The role of the parasitoids is to keep the budmoths at check so that these do not exhaust the foliage (PQI) and collapse the cycles. Thus, the more the parasitoid species, the longer does it take for the budmoth densities to reach their peak maxima and hence the longer the cycle lengths. The Tatras and the French Alps both have budmoth outbreaks in their larch forests. However, there are no cyclic outbreaks in the Tatras, while there are irregular cycles of all lengths in the French Alps. In both Tatras and the French Alps, larch trees grow sparser than in the Swiss Engadine valley. Sparseness in the Tatras is natural while in the French Alps it has resulted due to human intervention and this is not constant with time. The sparse growth of trees implies that the intraspecific competition between the budmoths is higher. The resulting low $c$ values produce no cycles or cycles with very small 
time periods [17]. When $c$ is too low, efficiency of the parasitoids fall, and cycles do not start at all, like in the Tatras. Numerically it is found that as $c$ increases, the time periods of the cycles increase. Biologically this makes sense as higher $c$ means more activity of the parasitoids leading to better check on the budmoth population which consequently takes a longer time to reach its peak values.

\section{Conclusion}

The larch forests in the French Alps differ from those in most other places on account of their being disturbed by human intervention and with consequent changes in the forest cover. The larch budmoth cycles here are irregular and in each case, the peak maxima stay on for as long as 2-5 years. From the analysis of cycles in the French Alps, it is very clear that not only is the intraspecific competition playing a vital role in the dynamics, but also the nature of the parasitoids that affect the dynamics. To check if different time periods and different durations of the maxima arise in the system, in this work we have proposed a 4-species tritrophic 5-dimensional model in which the parameter $c$ also varies with time. On simulating this model, we find that it is able to reproduce the observed time periods and also the observed durations of the maxima. From this we conclude that multiple parasitoid species in the French Alps which are experimentally known to infest a single budmoth play a vital role there.

\section{Acknowledgements}

JB and SVI acknowledge support from SERB, DST, Government of India (File No. SB/IR/NIAS/2016).

\section{References}

[1] C J Krebs, J. Mammalogy 77, 8 (1996)

[2] J Yoshimura, Am Nat. 149, 112 (1997)

[3] J Yoshimura, T Hayashi, Y Tanaka, K Tainaka and C Simon, Evolution 63, 288 (2008)

[4] P Turchin, L Oksanen, P Ekerholm, T Oksanen and H Henttonen, Nature 405, 562 (2000)

[5] F Bilodeau, G Gauthier and D Berteaux, Oecologia 172, 1007 (2013).

[6] J H Myers and J S Cory, Annu. Rev. Ecol. Evol. Syst. 44, 565 (2013)

[7] W Baltensweiler and A Fischlin, "The larch budmoth in the Alps" in Dynamics of forest insect populations pp. 331-351 (Springer, New York, 1988)

[8] A A Berryman, Trends. Ecol. Evol. 11, 28 (1996)
[9] J Esper, U Büntgen, D C Frank, D Nievergelt and A Liebhold, Proc. R. Soc. Lond. B 274, 671 (2007)

[10] W Baltensweiler, Oecologia 94, 62 (1993)

[11] O Konter, J Esper, A Liebhold, T Kyncl, L Schneider, E Düthorn and U Büntgen, Trends. Ecol. Evol. 29, 809 (2015)

[12] G Battipaglia, et al., Clim. Res. 62, 1 (2014)

[13] P Turchin, Complex population dynamics: A theoretical/empirical synthesis (Princeton University Press, NJ, 2003)

[14] V Delucchi, Entomophaga 27, 77 (1982)

[15] P Turchin, et al., Ecology 84, 1207 (2003)

[16] P Turchin, et al., "Population Cycles of the Larch Budmoth in Switzerland", in Population cycles: The case of trophic interactions, ed. A Berryman, pp.130-141 (Oxford University Press, 2002)

[17] S V Iyengar, J Balakrishnan and J Kurths, Sci. Rep 6 , 2785 (2016)

[18] S V Iyengar, J Balakrishnan and J Kurths, Chaos 26, 093111 (2016)

[19] S V Iyengar and J Balakrishnan,"q-deformations and the dynamics of the larch bud-moth population cycles" in Nature's longest threads: New Frontiers in the Mathematics and Physics of Information in Biology, eds. J Balakrishnan and B V Sreekantan, Ch. 8, 65-80 (World Scientific, Singapore, 2014)

[20] A J Nicholson and V A Bailey, J. Zool. 105, 551 (1935)

[21] C S Holling, Can. Entomol. 91, 385 (1959)

[22] C Tsallis, J. Stat. Phys. 52, 479 (1988)

[23] L Euler, Introduction in Analysis Infinitorum (Bousquet, Lausanne, 1748)

[24] E Heine, Handbuch der Kugelfunktionen (Reimer, Berlin, 1878), Vol. 1, reprinted by Physica-Verlag, Wurzburg (1961)

[25] F H Jackson, Proc. Roy. Soc. London 74, 64 (1904)

[26] F H Jackson, Trans. R. Soc. Edinburgh 46, 253 (1909)

[27] T Geisel and S Thomae, Phys. Rev. Lett. 52, 1936 (1984)

[28] R Jaganathan and S Sinha, Phys. Lett. A 338, 277 (2005)

[29] C Beck, E G D Cohen and S Rizzo, Europhys. News 36, 189 (2005)

[30] S Abe, U Tirnakli and P A Varotsos, Europhys. News 36, 206 (2005)

[31] A R Plastino and A Plastino, Phys. Lett. A 174, 384 (1993)

[32] G Kaniadakis, A Lavagno and P Quarati, Phys. Lett. B 369, 308 (1996)

[33] E Lutz, Phys. Rev. A 67, 051402(R) (2003)

[34] P Douglas, S Bergamini and F Renzoni, Phys. Rev. Lett. 96, 110601 (2006)

[35] Z-X Fan and A Braeuning, Ecol. Indicators 74, 160 (2017)

[36] C Hartl-Meier, J Esper, A Liebhold, O Konter, A Rothe and U Buentgen, Agr. Forest Entomol. (2017) DOI: 10.1111/afe. 12216

[37] N J Mills, Bull. Entomol. Res. 83, 103 (1993) 